\title{
Antikörper verbessert Motorik
}

Ein Antikörper gegen Myelin-assoziiertes Glykoprotein kann möglicherweise die neuronale Regeneration nach einem Schlaganfall beschleunigen. In einer Pilotstudie ließ sich die Gehgeschwindigkeit deutlich verbessern.

Neue Therapien, die auch noch Stunden nach einem Schlaganfall angewandt werden können und die langfristige Prognose verbessern, sind nötig, sagte Dr. Steven Cramer von der Universität in Irvine. Cramer stellte Daten zu einem neu entwickelten Antikörper (GSK24932) vor, der sich gegen Myelin-assoziiertes Glykoprotein (MAG) richtet. MAG wird nach einem Schlaganfall im Gehirn hochreguliert und verhindert, dass Nervenzellen neu aussprossen können. Neben Oligo-Myelin-Glykoprotein und NOGO-A zählt MAG zu den wichtigsten Nervenwachstumshemmern im Gehirn, sagte Cramer.

In der Phase-I/II-Studie erhielten 42 Patienten entweder 1, 5 oder $15 \mathrm{mg} / \mathrm{kgKG}$ des Antikörpers oder Placebo. Verabreicht wurde jeweils eine Dosis im Zeitfenster von 24-72 Stunden nach Beginn der Schlaganfallsymptome, eine weitere Dosis acht bis zehn Tage später. Voraussetzung für die Teilnahme an der Studie war zudem eine Arm- oder Beinbehinderung.

Beim Endpunkt „Gehgeschwindigkeit" schnitt die Verumgruppe deutlich besser ab als die Kontrollgruppe. Wurden die Ergebnisse aller drei Dosierungen zusammengefasst, war die Gehgeschwindigkeit sowohl nach einem Monat als auch nach drei und vier Monaten signifikant höher als mit Placebo.

Drei unerwünschte Ereignisse (ein Nierenversagen, ein erneuter Schlaganfall, eine Dysgeusie) wurden von den Studienärzten als möglicherweise therapieassoziiert bewertet. Acht Patienten entwickelten transient Antikörper gegen das Therapeutikum.

Der Wirkstoff wird nun in einer Phase-II-Studie bei über 160 Schlaganfallpatienten geprüft. Thomas Müller, Springer Medizin

American Academy of Neurology Annual Meeting, San Diego, 21.3.2013, Scientific Platform Session: Clinical aspects of ischemic stroke

\section{Kein Gefäßverschluss sichtbar? Dann bringt die Lyse wohl nichts}

\author{
Lässt sich bei Schlaganfallpatienten kein Gefäßver- \\ schluss in der Bildgebung nachweisen, scheint die Lyse \\ mit tPA nichts zu bringen. Auf die Lyse verzichten \\ wollen US-Neurologen hier aber trotzdem nicht.
}

Nicht selten sind Patienten mit klinischen Schlaganfallsymptomen in der Bildgebung unauffällig - ein Gefäßverschluss ist im MRT oder CT oft nicht erkennbar. Nützt ihnen dann eine Lyse mit tPA oder schadet sie mehr? Antwort darauf hat sich Dr. Sourabh Lahoti, Universität in Kentucky, aus der retrospektiven multizentrischen Analyse der Registerdaten von 200 Schlaganfallpatienten erhofft, bei denen in der Bildgebung kein arterieller Verschluss von Hirngefäßen festgestellt werden konnte.

65 von ihnen hatten eine Thrombolyse erhalten, die übrigen 135 nicht, zumeist, weil sie nicht mehr im Zeitfenster von viereinhalb Stunden für die tPA-Behandlung waren. Symptomstärke und Behinderungen lagen bei Klinikeinweisung in beiden Gruppen in etwa auf demselben Niveau. Nach 90 Tagen, so das Ergebnis der Analyse, gab es ebenfalls keine signifikanten Unterschiede: Im Schnitt erreichten $70 \%$ der Patienten in beiden Gruppen einen Wert auf der modified Rankin Scale (mRS) von 0-2 Punkten - sie hatten nach drei Monaten also keine gravierenden Behinderungen mehr. $4 \%$ der Patienten mit Lyse erlitten eine intrakranielle Blutung, ohne Lyse war dies bei keinem Pa- tienten der Fall. Die Mortalitätsrate war in der Gruppe mit Lyse etwas höher als bei den Patienten ohne tPA (6,3 versus 4,4\%), die Unterschiede waren nicht signifikant.

Anhand der Lokalisation und der Infarktgröße im diffusionsgewichteten MRT teilte man nun die Patienten in solche mit vermuteter spontaner Rekanalisation und solche mit lakunärem Infarkt, bei dem die Okklusion zu klein für den Nachweis ist, ein - beides Gründe, warum die Bildgebung keinen Verschluss zeigt. Auch hier gab es keine signifikanten Unterschiede beim funktionellen Ergebnis nach 3 Monaten, egal ob mit tPA behandelt oder nicht. Soll man nun Patienten ohne erkennbaren Gefäßverschluss in der Bildgebung überhaupt lysieren? Das gute funktionelle Ergebnis vor Augen, scheint die Lyse tatsächlich nichts zu bringen, sagte Lahoti. Andere Neurologen auf dem Kongress warnten jedoch vor voreiligen Schlüssen: Zum einen müssten die Ergebnisse erst in prospektiven Studien bestätigt werden, zum anderen verliere man wertvolle Zeit, wenn man immer erst auf die Ergebnisse der Bildgebung warten würde. Lahoti entgegnete, dass man per CT-Angiografie recht schnell ein Ergebnis erziele, regte aber ebenfalls größere prospektive Studien an, um den Nutzen oder Schaden der Lyse bei Patienten ohne arterielle Okklusion in der Bildgebung zu prüfen. Th.Müller, Springer Medizin

American Academy of Neurology Annual Meeting, San Diego, 21.3.2013, Scientific Platform Session: Clinical aspects of ischemic stroke 